\title{
The relationship between mixed vaginitis in late pregnancy and adverse pregnancy outcomes:a cross-sectional study.
}

\author{
huanrong li ${ }^{1}$, Mengting Dong ${ }^{1}$, Wenjuan Xie ${ }^{1}$, Wenhui Qi ${ }^{1}$, Fei Teng ${ }^{1}$, Huiyang $\operatorname{Li}^{1}$, Ye \\ Yan $^{1}$, Chen Wang ${ }^{1}$, Cha Han ${ }^{1}$, and Fengxia Xue ${ }^{1}$ \\ ${ }^{1}$ Tianjin Medical University General Hospital
}

March 28, 2021

\begin{abstract}
Objective:To clarify the epidemiological characteristics, risk factors, clinical symptoms and signs, laboratory features, and pregnancy outcomes of women with mixed vaginitis during late pregnancy. Methods:The study population consisted of 1103 women in late pregnancy who attended the Tianjin Medical University General Hospital from November 2019 to September 2020. Results:The incidence rate of mixed vaginitis during late pregnancy is $4.1 \%(44 / 1075)$. The independent risk factors for women with mixed vaginitis in late pregnancy are a positive glucose tolerance test ( $\mathrm{OR}=2.697,95 \%$ CI 1.293-5.625) and a history of vaginitis during pregnancy $(\mathrm{OR}=2.276,95 \%$ CI 1.030-5.032). Compared with women with single vaginitis, women with mixed vaginitis only have statistically significant differences in the yellow discharge ( $77.3 \%$ vs. $58.9 \%)$ and malodor (40.9\% vs. $22.7 \%)(\mathrm{P} \nmid 0.05)$, so they are easily confused with single vaginitis in clinical symptoms and signs. Compared with women with single vaginitis, women with mixed vaginitis have a significant difference in $\mathrm{pH}_{\llcorner} 4.5(72.7 \%$ vs. $36.2 \%)$ and $\mathrm{WBC} i 10 / \mathrm{hpf}$ $(81.8 \%$ vs. $38 \%)$ in laboratory examination $\left(\mathrm{P}_{\mathfrak{j}} 0.05\right)$. Therefore, laboratory diagnosis is the main method to distinguish single and mixed vaginitis. Compared with women without vaginitis, women with mixed vaginitis have an increased incidence of postpartum puerperal infections $\left(6.8 \%\right.$ vs. $1 \%$; $\left.\mathrm{P}_{\mathfrak{j}} 0.05\right)$. Conclusions:Mixed vaginitis in late pregnancy leads to an increased incidence of puerperal infections. Therefore, more attention should be paid to the early intervention of mixed vaginitis during pregnancy to prevent adverse pregnancy outcomes.
\end{abstract}

\section{Introduction}

Vaginitis is the most common infectious disease of female genital tract during childbearing age. Common vaginal infections mainly include BV (Bacterial Vaginosis), AV (Aerobic Vaginitis), VVC (Vulvovaginal Candidiasis), and TV (Trichomoniasis)(1). Mixed vaginitis refers to the infection of two or more pathogens in the vagina at the same time (2). Common mixed vaginitis mainly includes $\mathrm{BV}+\mathrm{VVC}, \mathrm{BV}+\mathrm{AV}, \mathrm{AV}+\mathrm{VVC}$, $\mathrm{TV}+\mathrm{AV}, \mathrm{TV}+\mathrm{BV}, \mathrm{TV}+\mathrm{VVC}$, etc. Existing studies have shown that the prevalence of mixed infections during pregnancy is $6.5 \%-13.2 \%(3-5)$, which is higher than that of $2.4 \%-6.4 \%$ in non-pregnant women( 6,7$)$. Low income, low education level, previous vaginal infections, poor personal hygiene, history of antibiotic use, and external hemorrhoids are known risk factors for BV, VVC and AV during pregnancy, but no studies have analyzed the risk factors of pregnant women with mixed vaginitis $(5,8)$. It is known that single vaginitis during pregnancy, such as $\mathrm{AV}, \mathrm{BV}$, and VVC, is associated with the increase in the incidence of PROM (premature rupture of membranes), premature delivery, etc $(1,5)$. The effect of mixed vaginitis on pregnancy outcome still needs further research. Whether the symptoms, signs and laboratory features of women with mixed vaginitis during late pregnancy are also confused with single vaginitis remains to be studied. Therefore, this study analyzed the mixed vaginitis in late pregnancy to summarize the epidemiological characteristics, risk factors, clinical symptoms and signs, laboratory characteristics, and their impact on adverse pregnancy outcomes in women with mixed vaginitis in the third trimester, so as to provide suggestions for clinical diagnosis and treatment. 


\section{Methods \\ Subjects}

We conducted a cross-sectional study on pregnant women in late pregnancy who had their first visit to the Department of Obstetrics and Gynecology, Tianjin Medical University General Hospital from November 2019 to July 2020. Inclusion criteria: 18-45 years old, intrauterine pregnancy, and intact fetal membrane. Exclusion criteria: unexplained vaginal bleeding; placenta previa; cervical cerclage; subjective induction of labor and abortion; antibiotic treatment within 4 weeks; sexual intercourse or use of vaginal medication within 3 days; mental or intellectual abnormalities or unable to answer related questions; incomplete questionnaire information; vulvar skin diseases, etc. The pregnant women were informed of the purpose of the study and all patients were included in the study after informed consent.

\section{Questionnaire}

The content of the questionnaire was asked by a professionally trained clinician. Each pregnant woman had a unique medical record number and the basic information, past medical history, symptoms of genital tract infection, signs of genital tract infection, fertile history, personal hygiene habits, and sexual experience were recorded through the questionnaire. Correction of gestational age based on ultrasound of first appearance of fetal heart and buds or last menstrual period.

\section{Vaginal examination, sampling and laboratory examination}

A professionally trained clinician will perform vaginal examination and collect vaginal secretions for pregnant women under bladder stones gesture and record the reproductive tract signs after physical examination in the questionnaire immediately. The doctor used a non-lubricated sterile speculum and a sterile long cotton swab to collect 3 swabs of vaginal secretions on the upper vaginal wall or posterior fornix. The secretions were immediately transferred to the laboratory for $\mathrm{pH}$ test, normal saline wet film phase contrast microscope (Olympus, Japan) $(400 \times)$ examination and Gram stain smear microscope $(1000 \times)$ examination for the diagnosis of $\mathrm{AV}, \mathrm{BV}, \mathrm{VVC}$, and $\mathrm{TV}$. Blind laboratory examinations were performed by two experienced and trained laboratory technicians, who did not know the clinical data.

\section{Diagnosis criteria}

The diagnosis of BV is based on the Nugent score. Under the microscope $(1000 \times)$, a quantitative score was made according to the number of the large Gram-staining positive bacilli, small Gram-staining variable bacilli, and Gram-stained variable curved bacilli, and the 7-10 score was divided into BV. Take the average of 10 high magnification fields under the microscope to score. The diagnostic criteria of AV referred to Donder's score(9). A quantitative score was made according to the proportion of lactobacilli, the number of white blood cells, the number of toxic white blood cells, the type of background bacteria, and the number of parabasal epithelial cells under the wet film microscope. The score [?]3 was divided into AV. The diagnosis of VVC is based on the hyphae and spore seen on the wet film and gram staining smear under microscope. TV diagnosis relies on normal saline wet film to see active Trichomonas vaginalis .

All pregnant women are aware of the vaginal discharge examination results and treated with AV, BV, TV, and VVC in accordance with the latest international guidelines and provided with health guidance and follow-up visits before delivery after treatment $(1,10)$.

\section{Study group assignment}

Grouping: The women were grouped according to different types of vaginitis (AV group, BV group, VVC group, TV group, $\mathrm{VVC}+\mathrm{AV}$ group, $\mathrm{VVC}+\mathrm{BV}$ group, $\mathrm{AV}+\mathrm{BV}$ group, and $\mathrm{AV}+\mathrm{TV}$ group) and normal women without vaginitis (Normal group). Among them, we totaled AV, BV, VVC, and TV groups into single vaginitis group and totaled $\mathrm{VVC}+\mathrm{AV}, \mathrm{VVC}+\mathrm{BV}, \mathrm{AV}+\mathrm{BV}$, and $\mathrm{AV}+\mathrm{TV}$ groups into mixed vaginitis group. And we compared the demographic characteristics, risk factors, clinical symptoms and signs, laboratory characteristics, and pregnancy outcomes of mixed vaginitis group with single vaginitis group and normal group. 


\section{Pregnancy outcomes}

Follow up the outcome of each pregnant woman's delivery. Preterm birth refers to the gestational age $<37$ weeks. Low birth weight refers to the birth weight of newborns $<2500 \mathrm{~g}$ regardless of gestational age. PROM refers to the rupture of the membranes before delivery. Neonatal asphyxia refers to: 1) there are risk factors for neonatal asphyxia; 2) Apgar score [?] 7 at 1 or 5 minutes after birth; 3) umbilical artery blood gas parameter $\mathrm{pH}<7.15 ; 4)$ exclude other causes of low Apgar score (11). Neonatal infection is defined as a laboratory-diagnosed bacterial infection (positive blood, cerebrospinal fluid, or urine culture) and/or the presence of clinical signs of infection (pneumonia, fever, hypothermia, or respiratory distress, etc.)(12). Stillbirth means that the fetus has no signs of life after birth at 28 weeks and later. Meconium-stained amniotic fluid (MSAF) means that amniotic fluid is contaminated with meconium and presents different colors, such as: lightly stained yellow, greenish color or dark green(13). Puerperal infection refers to infection of the genital tract during delivery and puerperium, causing local and systemic inflammatory, affecting postpartum recovery and even newborn feeding(14). Neonatal Intensive Care Unit (NICU) admission refers to the transfer of newborns to NICU after birth.

\section{Statistical methods}

SPSS 24.0 software was used for data processing and statistical analysis. Continuous variables were expressed as mean +- standard deviation. Categorical variables are represented by the Chi-square test. Dichotomous logistic regression was used to find the risk factors for mixed vaginitis, $\mathrm{AV}+\mathrm{BV}, \mathrm{VVC}+\mathrm{AV}$, and $\mathrm{VVC}+\mathrm{BV}$ groups. $P ¡ 0.05$ is considered statistically significant.

\section{Results}

\section{Prevalence of vaginitis}

A total of 1103 women were enrolled in the study, and 1075 women were finally enrolled according to the completeness of pregnancy outcome. Among 1075 women in the third trimester of pregnancy, 868 (80.7\%) women were normal without vaginitis, and $207(19.3 \%)$ women had vaginitis, including $163(15.2 \%)$ women with single vaginitis and $44(4.1 \%)$ women with mixed vaginitis. In women with single vaginitis, there were $88(8.2 \%)$ women with single VVC, $42(3.9 \%)$ women with single BV, 31(2.9\%) women with single $\mathrm{AV}$, and $2(0.2 \%)$ women with single TV. Among women with mixed vaginitis, there were $20(1.9 \%)$ women with $\mathrm{AV}+\mathrm{BV}, 11(1 \%)$ women with $\mathrm{VVC}+\mathrm{BV}, 12(1.1 \%)$ women with $\mathrm{VVC}+\mathrm{AV}$, and $1(0.1 \%)$ woman with $\mathrm{AV}+\mathrm{TV}($ Table S1).

\section{Characteristics of patients with mixed vaginitis}

Compared with women in normal group and women in corresponding single vaginitis group, women in $\mathrm{AV}+\mathrm{BV}$ group, $\mathrm{VVC}+\mathrm{BV}$ group, $\mathrm{VVC}+\mathrm{AV}$ group, and total mixed vaginitis group have no significant differences in age, educational level, gravidity, parity, and number of sexual partners $(P$ ¿0.05)(Table 1).

The positive rate of glucose tolerance test (25\% vs. $11.5 \%)$ and the incidence of vaginitis history during pregnancy ( $25 \%$ vs. $10.1 \%$ ) in women of mixed vaginitis group was higher than that in women of normal $\operatorname{group}(P \nmid 0.05)$.

The positive rate of glucose tolerance test ( $45.5 \% \mathrm{vs} .11 .5 \%)$, incidence of vaginitis history during pregnancy (45.5\% vs.10.1\%), and incidence of intercourse during pregnancy $(72.7 \%$ vs. $35 \%$ ) in women of VVC+BV group was higher than that in women of normal group $\left(P_{i} 0.05\right)$. The positive rate of glucose tolerance test in women of $\mathrm{VVC}+\mathrm{BV}$ group was higher than that in women of BV group $\left(45.5 \%\right.$ vs. $\left.9.5 \% ; P_{i} 0.05\right)$. The incidence of intercourse during pregnancy in women of $\mathrm{VVC}+\mathrm{BV}$ group was higher than that in women of VVC group (72.7\% vs. $33 \% ; P$ ¡0.05).

The history of reproductive tract infection before pregnancy in women of VVC+AV group was higher than that in women of VVC group (75\% vs.31.8\%), AV group ( $75 \%$ vs. $29 \%$ ) and normal group (75\%vs. $24.5 \%$ ) $(P\lceil 0.05)$. 


\section{Risk factors of women with mixed vaginitis}

Taking the women with normal group as a control, the positive glucose tolerance test during pregnancy (OR $=2.697,95 \%$ CI 1.293-5.625) and a history of vaginitis during pregnancy (OR $=2.276,95 \%$ CI $1.030-5.032)$ are independent risk factors leading to women suffering mixed vaginitis in late pregnancy (Table 2).

Positive glucose tolerance test during pregnancy ( $\mathrm{OR}=7.213$, 95\% CI 2.052-25.357), history of vaginitis during pregnancy $(\mathrm{OR}=6.384,95 \%$ CI 1.843-22.118), and intercourse during pregnancy $(\mathrm{OR}=5.047$, $95 \%$ CI 1.277-19.948) are independent risk factors that lead to women suffering VVC+BV vaginitis in late pregnancy.

\section{Clinical symptoms and signs of women with mixed vaginitis}

Compared with women in normal group, women in mixed vaginitis group, $\mathrm{AV}+\mathrm{BV}$ group, $\mathrm{VVC}+\mathrm{BV}$ group and $\mathrm{VVC}+\mathrm{AV}$ group showed significant differences in symptoms and signs $(P ; 0.05)$ (Table 3$)$. There are some differences in symptoms and signs between mixed vaginitis and corresponding single vaginitis.

Compared with patients in single vaginitis group, patients in mixed vaginitis group had obvious signs of yellow discharge $(77.3 \%$ vs.58.9\%) and malodor $(40.9 \%$ vs. $22.7 \%)(P ; 0.05)$. Compared with women in BV group, women in AV+BV group had obvious signs of yellow $(65 \%$ vs. $33.3 \%)$ and thick discharge $(35 \%$ vs.4.8\%) ( $P$;0.05). Compared with women with $\mathrm{BV}$, women with $\mathrm{VVC}+\mathrm{BV}$ vaginitis had obvious symptoms and signs of genital itching (72.7\% vs. $19 \%)$, vaginal erythema ( $63.6 \%$ vs. $26.2 \%)$, yellow ( $81.8 \%$ vs. $33.3 \%)$, and thick discharge $(45.5 \%$ vs.4.8\%) $(P ; 0.05)$. Compared with women with VVC, the symptoms and signs of genital burning ( $25 \%$ vs. $6.8 \%)$, vaginal erythema (91.7\% vs. $53.4 \%)$ and yellow discharge $(100 \%$ vs.64.8\%) in patients with $\mathrm{VVC}+\mathrm{AV}$ vaginitis are obvious $(P ; 0.05)$. Compared with women with $\mathrm{AV}$, the symptoms and signs of genital itching ( $75 \%$ vs. $6.5 \%)$, genital burning $(25 \%$ vs. $0 \%)$, redness and edema vulva ( $50 \%$ vs. $16.1 \%)$ and vaginal erythema $(91.7 \%$ vs. $38.7 \%)$ in patients with $\mathrm{VVC}+\mathrm{AV}$ vaginitis are obvious $(P \nmid 0.05)$.

It is not possible to distinguish single vaginitis from mixed vaginitis only from the symptoms and signs. Although there is a certain difference in symptoms and signs between the two, it is not sufficient as a specific indicator to distinguish the two. Such as yellow discharge or vaginal erythema is not unique to a certain vaginitis.

\section{Laboratory characteristics of women with mixed vaginitis}

Compared with women without vaginitis and women with single vaginitis, women with mixed vaginitis had a higher incidence of $\mathrm{pH}>4.5(72.7 \%$ vs. $6.9 \%$ and $72.7 \%$ vs.36.2\%) and $\mathrm{WBC}>10 / \mathrm{hpf}(81.8 \%$ vs. $26.2 \%$ and $81.8 \%$ vs. $38 \%)(P$ ¡0.05) (Table 3$)$.

Compared respectively with women in BV and normal groups, women in $\mathrm{AV}+\mathrm{BV}$ group had a higher incidence of $\mathrm{pH}>4.5(90 \%$ vs.57.1\% and $90 \%$ vs. $6.9 \%)$ and $\mathrm{WBC}>10 / \mathrm{hpf}(100 \%$ vs. $4.8 \%$ and $100 \%$ vs. $26.2 \%)(P$ i 0.05$)$. Compared with women in normal group and VVC group, women in VVC+BV vaginitis group had a higher incidence of $\mathrm{pH}>4.5(54.5 \%$ vs.6.9\% and $54.5 \%$ vs. $13.6 \%)(P$ ¡0.05). Compared with patients with BV vaginitis, patients with VVC+BV vaginitis had a higher incidence of WBC $>10 / \mathrm{hpf}(27.3 \%$ vs. $4.8 \%)(P ; 0.05)$. Compared with women in VVC and normal groups respectively, the incidence of $\mathrm{pH}>4.5(58.3 \%$ vs. $13.6 \%$ and $58.3 \%$ vs. $6.9 \%)$ and $\mathrm{WBC}>10 / \mathrm{hpf}(100 \%$ vs. $35.2 \%$ and $100 \%$ vs. $26.2 \%)$ of women in $\mathrm{VVC}+\mathrm{AV}$ group was higher $(P ; 0.05)$.

\section{Pregnancy outcomes of women with mixed vaginitis}

Puerperal infection is the main adverse outcome of women with mixed vaginitis. Compared with women without vaginitis $(6.8 \%$ vs.1.0\%) and single vaginitis $(6.8 \%$ vs. $1.2 \%)$, the incidence of puerperal infection in women with mixed vaginitis was higher $(P ; 0.05)$ (Table 4). Compared with women in VVC group (16.7\%vs. $0 \% ; P \nmid 0.05)$, AV group $(16.7 \%$ vs. $3.2 \%, P$ ¿ 0.05$)$ or normal group $(16.7 \%$ vs. $1 \%$; $P \nmid 0.05)$, the incidence of puerperal infection of women in VVC+AV group increased, and which was more significant between women with VVC+AV and women with VVC and between women with $\mathrm{VVC}+\mathrm{AV}$ and women in normal group. Compared with women in AV group (5\% vs. $3.2 \%$ ), BV group ( $5 \%$ vs. $2.4 \%$ ) or normal group (5\% vs. 
$1 \%$ ), the incidence of puerperal infection of women in AV+BV group increased, but the differences was not statistically significant $(P>0.05)$.

Mixed vaginitis may also lead to other adverse outcomes. Compared with women with single vaginitis, the incidence of MSAF $(22.7 \%$ vs.9.2\%; $P$ ¡ 0.05$)$ in patients with mixed vaginitis increased. Compared with women in VVC group, the incidence of PROM $(33.3 \%$ vs. $9.1 \% ; P ; 0.05)$ in women in VVC+AV group increased. Compared with patients in BV group, the incidence of NICU admission in patients in VVC+BV group was higher $(36.4 \%$ vs. $9.5 \% ; P\lceil 0.05)$.

\section{Discussion}

\section{Main Findings}

Pregnancy are known to be the main risk factors for Candida albicans infection (15). In vitro experiments confirmed that estrogen-pretreated mice with the same concentration of progesterone $\left(10^{-6} \mathrm{M}\right)$ in late pregnancy could strongly inhibit the anti-Candida activity of neutrophils, which led to an increase in the susceptibility of pregnant women to $\operatorname{VVC}(16)$. A study on the vagina of castrated rats showed that compared with rats not treated with estrogen, the vaginal microflora was more diverse in rats treated with estrogen and colonized with vaginal yeast(17), indicating that hormone status was involved in the characteristics of vaginal flora under Candida infection, which may also be the cause of mixed fungal and bacterial infection.

Diabetes is known to be the main risk factor for C.albicansinfection(15, 18). In 2018, Xinhong et al. (19) compared 186 gestational diabetes (GDM) patients with 200 healthy pregnant women and found that GDM not only increase the incidence of $\mathrm{VVC}$, but also increase the incidence of $\mathrm{BV}+\mathrm{VVC}$ mixed infection. Our study found that a positive glucose tolerance test during pregnancy is a risk factor leading to mixed VVC+BV infection, which is consistent with the above studies. However, another study did not find a difference in the incidence of BV and VVC between the diabetes group and the control group(20). Perhaps this study analyzed the difference in vaginitis during the first trimester.

In 2007, Britton et al.(20) studied the risk factors of BV in pregnancy and found that sexual intercourse during early pregnancy was associated with an increased risk of BV in the second trimester. Our research showed that sexual intercourse during pregnancy is a risk factor leading to VVC+BV in late pregnancy, which may be related to the fact that sexual intercourse itself has a greater interference effect on normal vaginal flora(21). Immunity during pregnancy is lower than during non-pregnancy, and the ability to recover the normal flora after sexual intercourse is reduced, which leads to the occurrence of mixed vaginitis.

Patients with mixed vaginitis have complex genital symptoms and signs(22). Our team previously analyzed the clinical symptoms, signs and laboratory characteristics of patients with AV mixed vaginitis(22). Similar to this research, our research found that patients with VVC+AV often had genital itching. Furthermore, our research also found that compared with patients with AV, the genital burning, redness and edema vulva and vaginal erythema were more obvious in patients with VVC+AV; compared with patients with VVC, the genital burning, vaginal erythema, and yellow discharge in patients with $\mathrm{VVC}+\mathrm{AV}$ were more obvious; and compared with patients with VVC, $\mathrm{pH}>4.5$ and $\mathrm{WBC}>10 / \mathrm{hpf}$ were significantly higher in patients with VVC+AV. Similar to this research, our research also found that compared with patients with BV, yellow vaginal discharge in patients with $\mathrm{AV}+\mathrm{BV}$ was more significant. However, our literature also found that compared with patients with $\mathrm{BV}, \mathrm{pH}>4.5$ and $\mathrm{WBC}>10 / \mathrm{hpf}$ were significantly higher in patients with $\mathrm{AV}+\mathrm{BV}$. Therefore, our research believes that mixed vaginitis has complex symptoms and signs, which is difficult to distinguish from the corresponding single vaginitis, and laboratory examination is the most effective method to distinguish mixed vaginitis from single vaginitis.

Mixed vaginitis is associated with adverse pregnancy outcomes. In 2018, Cha Han et al.(5) studied the pregnancy outcomes of pregnant women with AV and found that AV was associated with a high incidence of PROM. Our research found that the incidence of PROM increases in patients with VVC+AV compared with patients with VVC, which may be caused by AV. In 2016, Dingens et al.(23) studied the relationship between BV and adverse pregnancy outcomes in 12340 matched pregnant women and their 
newborns in Washington State. This research found that the incidence of neonatal ICU admission of BVpositive pregnant women increased. Our research finds that compared with patients with BV, the incidence of NICU admission is higher in patients with $\mathrm{VVC}+\mathrm{BV}$, which may be related to the increased incidence of NICU admission caused by VVC mixed vaginitis in the case of BV. Abnormal vaginal flora during pregnancy is associated with puerperal infection(19). In 2019, Wei Dai et al. (24)conducted a research on 380 women in late pregnancy and found that the puerperium infection was related to the colonization of Group B streptococcus (GBS) (AV pathogen) during pregnancy. Similar to our study, the VVC+AV mixed infection has an increased incidence of puerperal infection compared with the VVC group and the normal group, which may be related to AV. Analysis of the reason may be related to intrauterine infection caused by ascending infection of lower genital tract, activating intrauterine inflammation pathways, further causing puerperal infections(25-27).

\section{Strengths and Limitations}

However, there are still some problems in this study. For example, only the characteristics of women with mixed vaginitis in late pregnancy were studied, and the epidemiological characteristics of the first trimester and the second trimester were not studied. Although more than 1,000 women were included in this study, only 44 women had mixed vaginitis (20 women with $\mathrm{AV}+\mathrm{BV}, 11$ women with VVC+BV, 12 women with $\mathrm{VVC}+\mathrm{AV}$, and 1 woman with $\mathrm{AV}+\mathrm{TV}$ ). This study did not analyze the effect of treatment on pregnancy outcomes, mainly because the treatment methods of different mixed vaginitis are different. In the future, we plan to further expand the sample size to analyze the impact of mixed vaginitis and treatment of mixed vaginitis on pregnancy outcomes.

\section{Interpretation}

Mixed vaginitis refers to a vaginitis that combines two or more pathogens that cause AV, BV, VVC, and $\mathrm{TV}(2)$. The interaction between fungi and bacteria can mutually promote the colonization and virulence of pathogens (28). C.albicans (VVC pathogen) adhesion proteins, hyphal wall proteins, and transcriptional regulators play a crucial role in the interaction between C.albicans andStaphylococcus (AV pathogen $)(29,30)$. The mouse model of mixed infection of GBS and C.albicans confirmed that GBS could also promote the adhesion and colonization of C.albicans, while reducing the cellular immune response and host immune clearance rate of helper T cell 17 (Th17), ensuring the persistently chronic infection of the two(31). C.albicans can combine with a variety of streptococci and staphylococci(AV pathogen) to help them adhere and form biofilms(30). The dual-species biofilm formed can provide nutrients for pathogens to reproduce, and protect microorganisms from being eliminated by antimicrobial agents, further maintaining the pathogenic ability of pathogens(30, 32-37). The interaction between aerobic and anaerobic bacteria can promote biofilm formation and participate in the pathogenic process. In vitro experiments have confirmed thatEscherichia coli and Enterococcus faecalis (AV pathogens) coexist and interact with Gardnerella vaginalis (BV pathogen) to participate in the formation of BV biofilms (38). Therefore, the interaction between fungi and bacteria and between bacteria and bacteria is very important for the pathogenic processes of mixed vaginitis. Immunity is reduced during pregnancy. The host's ability to suppress pathogens is reduced. Mixed vaginitis during pregnancy may be more serious and complicated. The existence of multispecies biofilms in the vagina of pregnant women is related to the occurrence of adverse outcomes. This may come from the upward spread of biofilms, but the mechanism still needs further research.

\section{Conclusion}

Due to reduced immunity and increased disease susceptibility, mixed vaginitis is prone to occur during pregnancy. A positive glucose tolerance test during pregnancy and a history of vaginitis during pregnancy are risk factors for mixed vaginitis in late pregnancy. The symptoms and signs of women with mixed vaginitis during pregnancy are difficult to distinguish from women with simple vaginitis and are related to the adverse outcome of puerperal infection. It is recommended to efficiently distinguish mixed vaginitis through laboratory examination. Therefore, vaginitis should be diagnosed and treated during early pregnancy, and blood glucose management should be guided in time during pregnancy to prevent puerperal infection. 
Conflicts of Interest: The authors declare no conflict of interest.

Financial disclosure: The authors declare no financial disclosures.

Author' Contributions: All authors fulfilled the conditions required for authorship and have approved this submission. HL, CH, and FX conceived the study question, and all authors were involved in the study design. $\mathrm{HL}$, and YY were involved in the statistical analysis while HL, MD, and WX interpreted the results. HL created the first draft of the manuscript. MD, WX, WQ, FT, HL, and CW made substantial contributions to drafting the article and revising it critically. Furthermore, all authors have given their final approval for this version to be published and agree to be accountable for all aspects of the work.

Acknowledgements: Not applicable.

Ethics approval:This project was approved by the Ethics Committee of Tianjin Medical University General Hospital. (Ethical NO. IRB2020-WZ-194, 15 December 2020)

Funding: This work was supported by Tianjin Municipal Science and Technology Commission Special Foundation for Science and Technology Major Projects in Control and Prevention of Major Diseases (Grant No. 18ZXDBSY00200), General Project of the National Natural Science Foundation of China (Grant No. 82071674) and Tianjin Health Science and Technology Project (Grant No. KJ20003).

Supporting Information:Additional supporting information may be found online in the Supporting Information section at the end of the article.

Table 1. Comparison socio-demographic characteristics of mixed vaginitis with single vaginitis during pregnancy.

Table 2. Risk factors associated with mixed vaginitis during pregnancy.

Table 3. Comparison clinical symptoms and signs and laboratory characteristics of mixed vaginitis with single vaginitis during pregnancy.

Table 4. Comparison pregnancy outcomes of mixed vaginitis with single vaginitis.

Table S1. Prevalence of single and mixed vaginitis in pregnancy peoples.

\section{References}

1. Workowski KA, Bolan GA. Sexually transmitted diseases treatment guidelines, 2015. MMWR Recommendations and reports : Morbidity and mortality weekly report Recommendations and reports. 2015;64(Rr03):1-137.

2. Sobel JD, Subramanian C, Foxman B, Fairfax M, Gygax SE. Mixed vaginitis-more than coinfection and with therapeutic implications. Current infectious disease reports. 2013;15(2):104-8.

3. Kamara P, Hylton-Kong T, Brathwaite A, Del Rosario GR, Kristensen S, Patrick N, et al. Vaginal infections in pregnant women in Jamaica: prevalence and risk factors. International journal of STD \& AIDS. 2000;11(8):516-20.

4. Shrestha S, Tuladhar NR, Basnyat S, Acharya GP, Shrestha P, Kumar P. Prevalence of vaginitis among pregnant women attending Paropakar Maternity and Women's Hospital, Thapathali, Kathmandu, Nepal. Nepal Medical College journal : NMCJ. 2011;13(4):293-6.

5. Han C, Li H, Han L, Wang C, Yan Y, Qi W, et al. Aerobic vaginitis in late pregnancy and outcomes of pregnancy. European journal of clinical microbiology \& infectious diseases : official publication of the European Society of Clinical Microbiology. 2019;38(2):233-9.

6. Karaer A, Boylu M, Avsar AF. Vaginitis in Turkish women: symptoms, epidemiologic - microbiologic association. European journal of obstetrics, gynecology, and reproductive biology. 2005;121(2):211-5. 
7. Narayankhedkar A, Hodiwala A, Mane A. Clinicoetiological Characterization of Infectious Vaginitis amongst Women of Reproductive Age Group from Navi Mumbai, India. Journal of sexually transmitted diseases. 2015;2015:817092.

8. Konadu DG, Owusu-Ofori A, Yidana Z, Boadu F, Iddrisu LF, Adu-Gyasi D, et al. Prevalence of vulvovaginal candidiasis, bacterial vaginosis and trichomoniasis in pregnant women attending antenatal clinic in the middle belt of Ghana. BMC pregnancy and childbirth. 2019;19(1):341.

9. Donders GG, Vereecken A, Bosmans E, Dekeersmaecker A, Salembier G, Spitz B. Definition of a type of abnormal vaginal flora that is distinct from bacterial vaginosis: aerobic vaginitis. BJOG : an international journal of obstetrics and gynaecology. 2002;109(1):34-43.

10. Sherrard J, Wilson J, Donders G, Mendling W, Jensen JS. 2018 European (IUSTI/WHO) International Union against sexually transmitted infections (IUSTI) World Health Organisation (WHO) guideline on the management of vaginal discharge. International journal of STD \& AIDS. 2018;29(13):1258-72.

11. Chen ZL, Liu J. Interpretation of the Experts' Consensus on the criteria for the diagnosis and grading of neonatal asphyxia in China. Translational pediatrics. 2013;2(2):59-63.

12. Chan GJ, Lee AC, Baqui AH, Tan J, Black RE. Prevalence of early-onset neonatal infection among newborns of mothers with bacterial infection or colonization: a systematic review and meta-analysis. BMC infectious diseases. 2015;15:118.

13. Bouche C, Wiesenfeld U, Ronfani L, Simeone R, Bogatti P, Skerk K, et al. Meconium-stained amniotic fluid: a risk factor for postpartum hemorrhage. Therapeutics and clinical risk management. 2018;14:1671-5.

14. Song $\mathrm{H}, \mathrm{Hu} \mathrm{K}, \mathrm{Du} \mathrm{X}$, Zhang J, Zhao S. Risk factors, changes in serum inflammatory factors, and clinical prevention and control measures for puerperal infection. Journal of clinical laboratory analysis. 2020;34(3):e23047.

15. Sobel JD. Vulvovaginal candidosis. Lancet (London, England). 2007;369(9577):1961-71.

16. Nohmi T, Abe S, Dobashi K, Tansho S, Yamaguchi H. Suppression of anti-Candida activity of murine neutrophils by progesterone in vitro: a possible mechanism in pregnant women's vulnerability to vaginal candidiasis. Microbiology and immunology. 1995;39(6):405-9.

17. Larsen B, Galask RP. Influence of estrogen and normal flora on vaginal candidiasis in the rat. The Journal of reproductive medicine. 1984;29(12):863-8.

18. Ueta E, Osaki T, Yoneda K, Yamamoto T. Prevalence of diabetes mellitus in odontogenic infections and oral candidiasis: an analysis of neutrophil suppression. Journal of oral pathology \& medicine : official publication of the International Association of Oral Pathologists and the American Academy of Oral Pathology. 1993;22(4):168-74.

19. Zhang X, Liao Q, Wang F, Li D. Association of gestational diabetes mellitus and abnormal vaginal flora with adverse pregnancy outcomes. Medicine. 2018;97(34):e11891.

20. Trabert B, Misra DP. Risk factors for bacterial vaginosis during pregnancy among African American women. American journal of obstetrics and gynecology. 2007;197(5):477.e1-8.

21. Gajer P, Brotman RM, Bai G, Sakamoto J, Schutte UM, Zhong X, et al. Temporal dynamics of the human vaginal microbiota. Science translational medicine. 2012;4(132):132ra52.

22. Fan A, Yue Y, Geng N, Zhang H, Wang Y, Xue F. Aerobic vaginitis and mixed infections: comparison of clinical and laboratory findings. Archives of gynecology and obstetrics. 2013;287(2):329-35.

23. Dingens AS, Fairfortune TS, Reed S, Mitchell C. Bacterial vaginosis and adverse outcomes among full-term infants: a cohort study. BMC pregnancy and childbirth. 2016;16(1):278. 
24. Dai W, Zhang Y, Xu Y, Zhu M, Rong X, Zhong Q. The effect of group B streptococcus on maternal and infants' prognosis in Guizhou, China. Bioscience reports. 2019;39(12).

25. Lackritz EM, Wilson CB, Guttmacher AE, Howse JL, Engmann CM, Rubens CE, et al. A solution pathway for preterm birth: accelerating a priority research agenda. The Lancet Global health. 2013;1(6):e328-30.

26. Harrison MS, Eckert LO, Cutland C, Gravett M, Harper DM, McClure EM, et al. Pathways to preterm birth: Case definition and guidelines for data collection, analysis, and presentation of immunization safety data. Vaccine. 2016;34(49):6093-101.

27. Goldenberg RL, Hauth JC, Andrews WW. Intrauterine infection and preterm delivery. The New England journal of medicine. 2000;342(20):1500-7.

28. Cools P, Jespers V, Hardy L, Crucitti T, Delany-Moretlwe S, Mwaura M, et al. A Multi-Country CrossSectional Study of Vaginal Carriage of Group B Streptococci (GBS) and Escherichia coli in Resource-Poor Settings: Prevalences and Risk Factors. PloS one. 2016;11(1):e0148052.

29. Peters BM, Ovchinnikova ES, Krom BP, Schlecht LM, Zhou H, Hoyer LL, et al. Staphylococcus aureus adherence to Candida albicans hyphae is mediated by the hyphal adhesin Als3p. Microbiology (Reading, England). 2012;158(Pt 12):2975-86.

30. Lohse MB, Gulati M, Johnson AD, Nobile CJ. Development and regulation of single- and multi-species Candida albicans biofilms. Nature reviews Microbiology. 2018;16(1):19-31.

31. Yu XY, Fu F, Kong WN, Xuan QK, Wen DH, Chen XQ, et al. Streptococcus agalactiae Inhibits Candida albicans Hyphal Development and Diminishes Host Vaginal Mucosal TH17 Response. Frontiers in microbiology. 2018;9:198.

32. Forster TM, Mogavero S, Drager A, Graf K, Polke M, Jacobsen ID, et al. Enemies and brothers in arms: Candida albicans and gram-positive bacteria. Cellular microbiology. 2016;18(12):1709-15.

33. Harriott MM, Noverr MC. Ability of Candida albicans mutants to induce Staphylococcus aureus vancomycin resistance during polymicrobial biofilm formation. Antimicrobial agents and chemotherapy. 2010;54(9):3746-55.

34. Harriott MM, Noverr MC. Candida albicans and Staphylococcus aureus form polymicrobial biofilms: effects on antimicrobial resistance. Antimicrobial agents and chemotherapy. 2009;53(9):3914-22.

35. Fox EP, Cowley ES, Nobile CJ, Hartooni N, Newman DK, Johnson AD. Anaerobic bacteria grow within Candida albicans biofilms and induce biofilm formation in suspension cultures. Current biology : CB. 2014;24(20):2411-6.

36. Seth EC, Taga ME. Nutrient cross-feeding in the microbial world. Frontiers in microbiology. 2014;5:350.

37. Shrestha PM, Rotaru AE. Plugging in or going wireless: strategies for interspecies electron transfer. Frontiers in microbiology. 2014;5:237.

38. Castro J, Machado D, Cerca N. Escherichia coli and Enterococcus faecalis are able to incorporate and enhance a pre-formed Gardnerella vaginalis biofilm. Pathogens and disease. 2016;74(3).

Table 1 Comparison socio-demographic characteristics of mixed vaginitis with single vaginitis during pregnancy.

\begin{tabular}{|c|c|c|c|c|c|c|}
\hline & Normal & BV & $\mathrm{AV}$ & VVC & $\mathrm{TV}$ & $\mathrm{AV}+\mathrm{BV}$ \\
\hline & $\mathrm{n}(\%)$ & $\mathrm{n}(\%)$ & $\mathrm{n}(\%)$ & $\mathrm{n}(\%)$ & $\mathrm{n}(\%)$ & $\mathrm{n}(\%)$ \\
\hline $\begin{array}{l}\text { Age, years } \\
\text { ¡30 } \\
30[?] \text {;35 }\end{array}$ & $\begin{array}{l}240(27.6) \\
367(42.3)\end{array}$ & $\begin{array}{l}10(23.8) \\
20(47.6)\end{array}$ & $\begin{array}{l}9(29) \\
15(48.4)\end{array}$ & $\begin{array}{l}30(34.1) \\
37(42)\end{array}$ & $\begin{array}{l}2(100) \\
0\end{array}$ & $\begin{array}{l}5(25) \\
8(40)\end{array}$ \\
\hline
\end{tabular}




\begin{tabular}{|c|c|c|c|c|c|c|}
\hline & Normal & BV & AV & VVC & TV & $\mathrm{AV}+\mathrm{BV}$ \\
\hline 35 & $261(30.1)$ & $12(28.6)$ & $7(22.6)$ & $21(23.9)$ & 0 & $7(35)$ \\
\hline \multicolumn{7}{|l|}{ Educational status } \\
\hline junior high school & $36(4.1)$ & $2(4.8)$ & $2(6.5)$ & $5(5.7)$ & $1(50)$ & 0 \\
\hline senior high school[?];college & $213(24.5)$ & $11(26.2)$ & $3(9.7)$ & $25(28.4)$ & $1(50)$ & $2(10)$ \\
\hline college or above & $619(71.3)$ & $29(69)$ & $26(83.9)$ & $58(65.9)$ & 0 & $18(90)$ \\
\hline \multicolumn{7}{|l|}{ Gravidity } \\
\hline 1 & $380(43.8)$ & $16(38.1)$ & $12(38.7)$ & $41(46.6)$ & $2(100)$ & $10(50)$ \\
\hline $2-3$ & $386(44.5)$ & $23(54.8)$ & $18(58.1)$ & $39(44.3)$ & 0 & $9(45)$ \\
\hline 4 & $102(11.8)$ & $3(7.1)$ & $1(3.2)$ & $8(9.1)$ & 0 & $1(5)$ \\
\hline \multicolumn{7}{|l|}{ Parity } \\
\hline 0 & $564(65)$ & $31(73.8)$ & $29(64.5)$ & $65(73.9)$ & $2(100)$ & $15(75)$ \\
\hline 1 & $282(32.5)$ & $11(26.2)$ & $9(29)$ & $21(23.9)$ & 0 & $5(25)$ \\
\hline 2 & $22(2.5)$ & 0 & $2(6.5)$ & $2(2.3)$ & 0 & 0 \\
\hline \multicolumn{7}{|l|}{ Abortion } \\
\hline 0 & $515(59.3)$ & $21(50)$ & $20(64.5)$ & $49(55.7)$ & $2(100)$ & $16(80)$ \\
\hline 1 & $208(24)$ & $16(38.1)$ & $9(29)$ & $30(34.1)$ & 0 & $1(5)$ \\
\hline 2 & $145(16.7)$ & $5(11.9)$ & $2(6.5)$ & $9(10.2)$ & 0 & $3(15)$ \\
\hline \multicolumn{7}{|l|}{ Number of sexual partners } \\
\hline 1 & $718(82.7)$ & $32(76.2)$ & $27(87.1)$ & $75(85.2)$ & $1(50)$ & $16(80)$ \\
\hline $2-3$ & $144(16.6)$ & $9(21.4)$ & $4(12.9)$ & $13(14.8)$ & $1(50)$ & $3(15)$ \\
\hline 4 & $6(0.7)$ & $1(2.4)$ & 0 & 0 & 0 & $1(5)$ \\
\hline Positive of oral glucose tolerance test during pregnancy & $100(11.5)$ & $4(9.5)$ & $5(16.1)$ & $19(21.6)$ & $1(50)$ & $4(20)$ \\
\hline History of vaginitis during pregnancy & $88(10.1)$ & $9(21.4)$ & $4(12.9)$ & $22(25)$ & $1(50)$ & $4(20)$ \\
\hline Sex during pregnancy & $304(35)$ & $18(42.9)$ & $5(16.1)$ & $29(33)$ & $2(100)$ & $8(40)$ \\
\hline History of genital infections before pregnancy & $213(24.5)$ & $9(21.4)$ & $9(29)$ & $28(31.8)$ & 0 & $3(15)$ \\
\hline Panty liner use & $151(17.4)$ & $5(11.9)$ & $6(19.4)$ & $17(19.3)$ & $1(50)$ & $3(15)$ \\
\hline
\end{tabular}

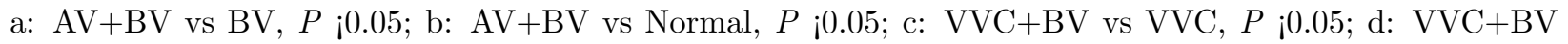
vs $\mathrm{BV}, P$ ¡ 0.05 ; e: VVC+BV vs Normal, $P$ i0.05; f: VVC + AV vs VVC, $P$ i 0.05 ; g: VVC + AV vs $\mathrm{AV}, P$ $¡ 0.05 ; \mathrm{h}: \mathrm{VVC}+\mathrm{AV}$ vs Normal, $P \nmid 0.05 ; \mathrm{i}$ : Mixed vaginitis vs Normal, $P \nmid 0.05 ; \mathrm{j}$ : Mixed vaginitis vs Single Vaginitis, $P$ ¡0.05.

Table 2 Risk factors associated with mixed vaginitis during pregnancy.

\begin{tabular}{|c|c|c|c|}
\hline \multicolumn{2}{|l|}{ Risk factor } & \multirow{2}{*}{$\begin{array}{l}\text { Mixed vaginitis vs Normal } \\
\text { Univariate analysis }\end{array}$} & \multirow{2}{*}{ Mixed vaginitis vs Normal } \\
\hline & & & \\
\hline & & OR $(95 \% \mathrm{CI})$ & P Value \\
\hline \multirow[t]{2}{*}{ Positive of oral glucose tolerance test during pregnancy } & No & 1 & 0.01 \\
\hline & Yes & $2.560(1.254-5.225)$ & \\
\hline \multirow[t]{2}{*}{ History of vaginitis during pregnancy } & No & 1 & 0.003 \\
\hline & Yes & $2.955(1.442-6.052)$ & \\
\hline \multirow[t]{2}{*}{ Sex during pregnancy } & No & 1 & 0.161 \\
\hline & Yes & $1.546(0.840-2.844)$ & \\
\hline \multirow[t]{2}{*}{ History of genital infections before pregnancy } & No & 1 & 0.278 \\
\hline & Yes & $1.435(0.747-2.757)$ & \\
\hline \multirow[t]{2}{*}{ Panty liner use } & No & 1 & 0.893 \\
\hline & Yes & $1.055(0.481-2.316)$ & \\
\hline
\end{tabular}


Table 3 Comparison clinical symptoms and signs and laboratory characteristics of mixed vaginitis with single vaginitis during pregnancy.

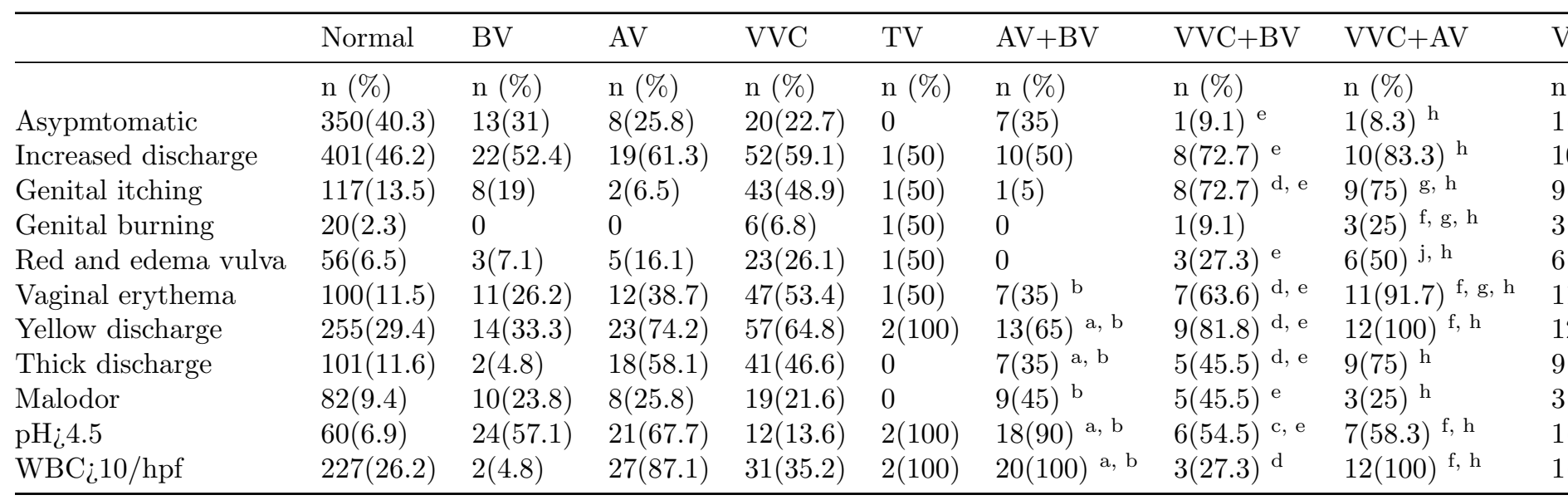

a: $\mathrm{AV}+\mathrm{BV}$ vs BV,$P$ 0.05 ; $\mathrm{b}: \mathrm{AV}+\mathrm{BV}$ vs Normal, $P$ ¡0.05; c: VVC+BV vs VVC, $P$ ¡0.05; d: VVC+BV vs $\mathrm{BV}, P$ ¡0.05; e: VVC+BV vs Normal, $P$ ¡0.05; f: VVC+AV vs VVC, $P$ ¡ 0.05 ; g: VVC+AV vs AV, $P$ $¡ 0.05 ; \mathrm{h}: \mathrm{VVC}+\mathrm{AV}$ vs Normal, $P \nmid 0.05 ; \mathrm{i}$ : Mixed vaginitis vs Normal, $P$ ¡0.05; j: Mixed vaginitis vs Single Vaginitis, $P$ ¡0.05.

Table 4 Comparison pregnancy outcomes of mixed vaginitis with single vaginitis.

\begin{tabular}{|c|c|c|c|c|c|c|c|c|}
\hline Outcomes & Normal & BV & $\mathrm{AV}$ & VVC & $\mathrm{TV}$ & $\mathrm{AV}+\mathrm{BV}$ & $\mathrm{VVC}+\mathrm{BV}$ & $v$ \\
\hline & $\mathrm{n}(\%)$ & $\mathrm{n}(\%)$ & $\mathrm{n}(\%)$ & $\mathrm{n}(\%)$ & $\mathrm{n}(\%)$ & $\mathrm{n}(\%)$ & $\mathrm{n}(\%)$ & $\mathrm{n}$ \\
\hline \multicolumn{9}{|l|}{ Delivery mode } \\
\hline Vaginal delivery & $368(42.4)$ & $19(45.2)$ & $17(54.8)$ & $40(45.5)$ & $1(50)$ & $10(50)$ & $6(54.5)$ & \\
\hline Ceasarean section & $500(57.6)$ & $23(54.8)$ & $14(45.2)$ & $48(54.5)$ & $1(50)$ & $10(50)$ & $5(45.5)$ & \\
\hline PROM & $149(17.2)$ & $8(19)$ & $10(32.3)$ & $8(9.1)$ & $1(50)$ & $2(10)$ & $1(9.1)$ & \\
\hline Preterm & $62(7.1)$ & $3(7.1)$ & $2(6.5)$ & $9(10.2)$ & 0 & 0 & 0 & \\
\hline Stillbirth & $2(0.2)$ & 0 & 0 & 0 & 0 & 0 & 0 & 0 \\
\hline Puerperal infection & $9(1)$ & $1(2.4)$ & $1(3.2)$ & 0 & 0 & $1(5)$ & 0 & \\
\hline Neonatal infection & $88(10.1)$ & $2(4.8)$ & $5(16.1)$ & $15(17)$ & 0 & $1(5)$ & $2(18.2)$ & \\
\hline Meconium-stained amniotic fluid (MSAF) & $90(10.4)$ & $3(7.1)$ & $4(12.9)$ & $8(9.1)$ & 0 & $3(15)$ & $3(27.3)$ & \\
\hline Low birth weight & $46(5.3)$ & $3(7.1)$ & $1(3.2)$ & $6(6.8)$ & 0 & 0 & $1(9.1)$ & \\
\hline Neonatal asphyxia & $10(1.2)$ & $1(2.4)$ & 0 & 0 & 0 & $1(5)$ & 0 & \\
\hline NICU admission & $147(16.9)$ & $4(9.5)$ & $5(16.1)$ & $18(20.5)$ & 0 & $1(5)$ & $4(36.4)^{\mathrm{d}}$ & \\
\hline
\end{tabular}

a: $\mathrm{AV}+\mathrm{BV}$ vs $\mathrm{BV}, P\lceil 0.05$; b: $\mathrm{AV}+\mathrm{BV}$ vs Normal, $P$ ¡0.05; c: VVC+BV vs VVC, $P$ ¡0.05; d: VVC $+\mathrm{BV}$ vs $\mathrm{BV}, P$ ¡0.05; e: VVC+BV vs Normal, $P$ ¡0.05; f: VVC+AV vs VVC, $P$ ¡ 0.05 ; g: VVC+AV vs AV, $P$ $¡ 0.05$; h: VVC + AV vs Normal, $P \nmid 0.05$; i: Mixed vaginitis vs Normal, $P \nmid 0.05 ; j$ : Mixed vaginitis vs Single Vaginitis, $P \nmid 0.05$. 